\title{
The Expanding World of DNA and RNA
}

Tingjian Chen, Narupat Hongdilokkul, Zhixia Liu, Deepak Thirunavukarasu, Floyd E. Romesberg*

Department of Chemistry, The Scripps Research Institute, 10550 North Torrey Pines Road, La Jolla, California USA

*Corresponding author. Tel.: +1 (858) 784 7290; fax: +1 (858) 7847472.

E-mail address: floyd@scripps.edu (F.E. Romesberg)

\begin{abstract}
DNA and RNA are remarkable because they can both encode information and possess desired properties, including the ability to bind specific targets or catalyze specific reactions. Nucleotide modifications that do not interfere with enzymatic synthesis are now being used to bestow DNA or RNA with properties that further increase their utility, including phosphate and sugar modifications that increase nuclease resistance, nucleobase modifications that increase the range of activities possible, and even whole nucleobase replacement that results in selective pairing and the creation of unnatural base pairs that increase the information content. These modifications are increasingly being applied both in vitro and in vivo, including in efforts to create semi-synthetic organisms with altered or expanded genetic alphabets.
\end{abstract}




\section{Introduction}

The template-directed enzymatic synthesis of DNA and RNA makes them unique among all materials and allows them to mediate the heritable storage and retrieval of biological information. The in vitro reconstitution of these processes has revolutionized biotechnology, enabling applications ranging from sequencing and cloning to a myriad of emerging techniques based on the genome-wide analysis of DNA and RNA. When combined with the range of structures available to single-stranded DNA and RNA, which allows them to recognize specific targets (aptamers) and even catalyze reactions, these processes allow for the laboratory evolution of functional oligonucleotides (or SELEX: systematic evolution of ligands by exponential enrichment) for applications ranging from affinity reagents and diagnostics to therapeutics. Finally, the emerging field of synthetic biology takes advantage of enzymatic synthesis in vitro and in vivo to create "parts" that may be used to impart living cells with new attributes or functions.

Despite these revolutionary applications, the limited composition (natural nucleotides), stability to nucleases, and range of physicochemical properties of natural DNA and RNA limit their potential. This has spurred the search for modified, and even wholly unnatural nucleotides, that bestow the corresponding oligonucleotides with desired properties but that remain compatible with enzymatic synthesis. For example, while the canonical nucleobases are capable of stacking, the types of motifs accessible are limited by the associated hydrophilic hydrogen-bond donors and acceptors, and thus DNA and RNA are largely unable to access many of the types of hydrophobic cores and surfaces that play such an essential role in protein folding and function.

In this review we focus on two types of nucleotides, natural nucleotides that are modified for improved properties (stability or function) and nucleotides that have wholly unnatural 
nucleobases that mediate their selective pairing and that thus constitute unnatural base pairs (UBPs) with the potential to expand the compositional and thereby the informational content of DNA. We first summarize the progress made toward the identification and use of such nucleotides in vitro, with an emphasis on SELEX applications and the development of UBPs. We then provide a survey of recent progress toward the exciting goal of using modified nucleotides and UBPs in vivo as the basis of semi-synthetic organisms.

\section{SELEX with modified RNA and DNA}

Initially, SELEX was developed with RNA, which requires transcription of a DNA library prior to the selection step as well as reverse transcription back to DNA afterward to enable amplification of the selected sequences (Figure 1a). The enzymes commonly employed are the RNA polymerase from T7 bacteriophage (T7 RNAP) and Superscript ${ }^{\mathrm{TM}}$ or Superscript II ${ }^{\mathrm{TM}}$ reverse transcriptases. In principle, the use of DNA streamlines the SELEX protocol by requiring a single enzymatic step (Figure 1b). However, with modified RNA or DNA this requires the direct amplification of the modified oligonucleotides, which is generally challenging. Moreover, inefficient amplification is likely to introduce significant sequence biases that may result in the unwanted enrichment of more easily amplified sequences. Thus, it is common to use a protocol for DNA SELEX that is analogous to that of RNA SELEX: the DNA is first "transcribed" by a DNA polymerase using modified dNTPs, and then after selection, "reverse transcribed" by a DNA polymerase back into natural DNA for the more challenging amplification step. The DNA polymerases that appear most able to tolerate modifications include KOD Dash, Vent (exo-), Phusion, and Therminator. 
Particularly with RNA, efforts have been directed toward the use of modified nucleotides that impart their corresponding oligonucleotides with resistance to nuclease-mediated degradation, including replacement of the non-bridging oxygen atom within the phosphate backbone with sulfur ( $\alpha$-thio) or $\mathrm{BH}_{3}$ ( $\alpha$-borane), or modification of the sugar with 4'-thio or 2'-amino $\left(\mathrm{NH}_{2}\right)$, fluoro $(\mathrm{F})$, or methoxy $(\mathrm{OMe})$ substituents. Modifications at both sugar positions are best tolerated with pyrimidines, and as a compromise between stability and the efficiency of synthesis, mixed libraries of modified pyrimidines and natural purines are commonly employed. 2'-F substituents have been used the most (reviewed in [1]), and 2'-F-pyrimidine-modified libraries have recently been subjected to in vivo selection for stable aptamers targeting tumor cells in living animals [2] or that penetrate the blood-brain barrier [3], and the Soh group has recently demonstrated the utility of including selection pressure for specificity to identify 2'-Fpyrimidine-modified RNA aptamers that recognize different subunits of integrin $\alpha \mathrm{V} \beta 3$ [4]. Optimized conditions and the use of the Y639F/H784A double mutant of T7 RNAP has also enabled the transcription of oligonucleotides with increased levels of 2'-OMe substitutions, which have been used for the identification of stabilized aptamers against vascular endothelial growth factor (VEGF) [5] and staphylococcal protein A [6]. The recent discovery of additional T7 RNAP mutants that better recognize 2'-OMe substrates $[7,8]$ and a mutant of the Syn5 RNA polymerase from cyanophage Syn5 that better recognizes 2'-F substrates [9] should facilitate the identification of stabilized aptamers in the future.

Traditionally, efforts to employ SELEX with sugar-modified DNA have been less common, however, a DNA aptamer containing 2'-F dG that binds thrombin has recently been disclosed [10]. In addition, we recently reported the directed evolution of a variant 
of Stoffel fragment (the catalytic subunit of Taq DNA polymerase), SFM4-3, that efficiently PCR amplifies DNA partially modified with 2'-F or 2'-OMe substituents, including purines and/or pyrimidines [11]. An increasingly diverse range of sugar modified nucleotides that are recognized by wild type or mutant DNA polymerases has recently attracted attention, including threose nucleic acids (TNA), hexitol nucleic acids (HNA), cyclohexenyl nucleic acids (CeNA), locked nucleic acids (LNA), arabinonucleic acids (ANA), and 2'-fluoro-arabinonucleic acids (FANA) (Figure 2a). For example, Chaput and co-workers used a variant of SELEX known as SELMA (SELection with Modified Aptamers [12,13]) to select TNA aptamers that bind thrombin [14]. Therminator DNA polymerase was used to extend a DNA hairpin primer/template with the modified nucleotides, permitting amplification of the DNA primer-template after selection and resynthesis of the selected TNA aptamer. Kuwahara and co-workers used a mixture of mutant and/or wild type KOD DNA polymerases to "transcribe" and "reverse transcribe" mixed LNA/DNA oligonucleotides during selection of an anti-thrombin aptamer $[15,16]$. Holliger and co-workers have dramatically expanded the potential of these analogs by evolving a series of TgoT DNA polymerase mutants to better "transcribe" and "reverse transcribe" these modified nucleotides, and they have used them to select HNA aptamers targeting HIV trans-activating response RNA and hen egg lysozyme [17•], a FANA aptamer targeting HIV-1 RT [18], and catalytic nucleic acids containing ANA, FANA, HNA, and CeNA moieties [19]. In addition, we have evolved several mutants of the Stoffel fragment that better "transcribe" and "reverse transcribe" fully modified 2'-F and 2'-OMe oligonucleotides [11].

To increase the physicochemical potential of oligonucleotides, efforts have been directed toward the use of nucleotides with modified nucleobases, and while modification at the 5-position of pyrimidines is best tolerated, modification at the 7-position of deazapurines 
is also possible. It is useful to consider the modifications as being composed of "linkers," to which the polymerase is very sensitive, and attached functionalities, to which the polymerase is generally less sensitive. This allows for a significant simplification of the literature as most modifications are based on one of five linker groups, the most common of which consist of several subgroups (Figure 2b). Far from being inert, these linkers mediate interactions with the polymerase during incorporation [20] and within the oligonucleotide once incorporated $[21 \cdot \bullet]$. To expand the range of modifications that are compatible with enzymatic synthesis, Mayer and coworkers recently demonstrated that after PCR-mediated incorporation of C5-ethynyl-dU, the ethynyl groups may be modified via Click chemistry, allowing access to a wide range of triazole-linked functionalities [22•].

While it is clear that the backbone and sugar modifications achieve the goal of increasing stability, whether nucleobase modifications actually increase the range of potential functions is less clear. This question has been elegantly addressed by SomaLogic Inc., who has structurally characterized three "SOMAmers" (Slow Off-rate Modified Aptamers) which were selected against PDGF [23], IL-6 [24] or NGF [25] and where each $\mathrm{dT}$ is replaced by $\mathrm{dU}$ analogs with simple aromatic rings (typically benzyl rings) attached to the 5 position via carboxamide linkers. Mutational analysis revealed that the 8 to 10 appended aromatic rings in each SOMAmer are generally required for binding, and structural analysis revealed that they nucleate the formation of clusters that present optimal surfaces for target recognition or that act as hydrophobic cores that support unique structure formation (Figure 3). Two particularly remarkable motifs observed were a four-ring stack in the IL-6 SOMAmer (with one ring provided by a tyrosine side chain from the protein; Figure 3e) and two "benzyl-dU zippers" in the NGF SOMAmer, where the benzyl group of one modified nucleotide packs with the uridine 
ring of the other (Figure $3 f$ ). These motifs demonstrate the potential of augmenting the repertoire of forces naturally available to nucleic acids with hydrophobic and packing forces, and are reminiscent of interactions common to proteins, as well as to the mode of pairing observed with predominantly hydrophobic unnatural base pairs (see below).

\section{UBPs and their in vitro applications}

While modification of the natural nucleotides promises to stabilize and expand the potential functions available to oligonucleotides, the development of a UBP that functions along side the natural base pairs promises to expand their information content. Three families of UBPs have been extensively validated and are exemplified by $d \mathbf{Z}-\mathrm{dP}$, developed by the Benner laboratory, dDs-dPx, developed by the Hirao laboratory, and dNaM-d5SICS, developed by our laboratory [26-28] (Figure 4a). The first of these UBPs is based on a hydrogen bonding pattern that is orthogonal to those employed by the natural base pairs, while the latter two are based on hydrophobic and packing forces. When incorporated into DNA, each may be efficiently replicated and transcribed into RNA.

The structures of dNaM-d5SICS and several derivatives have been determined within duplex DNA, and the unnatural nucleobases are found to pair via cross-strand intercalation [29] (Figure 4b). This accounts for their stability, but is inconsistent with their efficient replication by polymerases, which is based on the recognition of WatsonCrick-like structures. However, the structure of the UBP formed by pairing an unnatural triphosphate with its cognate nucleotide in a template within the active site of a DNA polymerase revealed a Watson-Crick-like, planar edge-to-edge structure (Figure 4c) [29]. Thus, the dNaM-d5SICS family of UBPs (and perhaps dDs-dPx as well) is stable and efficiently replicated because the hydrophobic and packing forces that mediate 
pairing are strong but sufficiently plastic to adopt to their duplex or polymerase environment. Moreover, this mode of interaction has been observed with other hydrophobic nucleobase analogs [30-33] and with the hydrophobic aromatic rings of SOMAmers (see above), which suggests that it is robust and easily accessed with modified nucleotides.

DNA containing the dDs-dPx or $d Z-d P$ UBPs has recently been used to evolve aptamers with novel properties. The Hirao group used a modification of the $\mathrm{dDs}$ - $\mathrm{dPx}$ UBP to evolve aptamers that use the unique functionality provided by dDs to recognize VEGF165 or IFN-y [34•], and the Benner group used libraries containing the unnatural nucleotides $d \mathbf{P}$ and $d \mathbf{Z}$ to evolve aptamers that bind breast or liver cancer cells $[35,36]$. In each case the unnatural nucleotides are required for binding.

Along with linkers akin to those used to modify natural nucleotides, the UBPs allow for the site-specific attachment of functionality within an amplifiable context, and the use of dDs-dPx and dNaM-d5SICS to array small molecules or proteins with unprecedented spatial resolution has been demonstrated $[37,38]$. In addition, we also recently reported the use of a derivative of the dNaM-d5SICS UBP to direct the transcription of the central domain of Thermus thermophilus $16 \mathrm{~S}$ ribosomal RNA site-specifically labeled with fluorophores at two positions, which with single molecule FRET characterization revealed the existence of previously unknown assembly complexity [39].

\section{Reading and writing modified oligonucleotides in vivo}

An increasingly central goal in the field is to use modified oligonucleotides or UBPs within cells to control, alter, or augment their physiology. Towards this goal, several groups have reported the successful conversion of modified DNA into natural DNA in 
vivo. Kool and co-workers demonstrated that a short stretch of DNA with size-expanded analogs of natural nucleobases can be copied into their natural counterparts in living $E$. coli cells [40], and Marliére and co-workers showed that a short stretch of CeNA, ANA, or HNA can be converted into DNA [41]. Most recently, the Matsuda group demonstrated that 4'-thio-DNA can be transcribed into RNA in mammalian cells [42].

The production of modified nucleic acids in vivo is more challenging, as the modified triphosphates must be made available and must be selectively utilized by polymerases. The replication of modified DNA in vivo was first demonstrated by Marliére and coworkers in 2011 when they showed that the 5'-chlorouracil analog of dT could replace the majority of $\mathrm{dT}$ nucleotides in the genome of $E$. coli cells [43••]. The triphosphate was made available by providing 5'-chlorouracil to the growth medium and expressing a deoxyribosyltransferase from Lactobacillus leichmannii. Competition with dTTP was reduced by disabling its major route of biosynthesis, and the efficiency of unnatural triphosphate use was optimized by adaptive evolution.

The deployment of a UBP in a living cell would allow for the creation of semi-synthetic organisms that store (and eventually retrieve) increased information. Following the validation of dNaM-d5SICS in vitro, we set out to test whether DNA containing this UBP can be replicated within E. coli cells [44••]. The first challenge of getting the unnatural triphosphates into the cells was solved by the expression of an algal nucleoside triphosphate transporter. Cells expressing the transporter were then transformed with a plasmid containing the UBP and then grown in media supplemented with dNaMTP and d5SICSTP. Analyses of the plasmid after various periods of growth revealed that not only was the UBP retained, the fidelity of replication (retention per doubling) was greater 
than $99.4 \%$. This modified E. coli is the first semi-synthetic organism able to store increased information in its DNA (Figure 5).

\section{Conclusions}

DNA and RNA are remarkable molecules and our ability to manipulate them has impacted virtually every area of the biological sciences. The increasing ability to efficiently synthesize backbone- or sugar-modified oligonucleotides has allowed for the discovery of aptamers against a variety of proteins and small molecules that are stable to nuclease-mediated degradation. Recent successes in the directed evolution of polymerases tailored for the modified substrates should now accelerate progress.

While too few studies have focused on if and how nucleobase modifications allow access to an increased range of function, comparative analysis of SELEX experiments suggest that they increase the likelihood of aptamer identification, and structural analyses suggest that they do so by allowing oligonucleotides to adopt a broader range of conformations and structural motifs $[21 \bullet \bullet, 45,46]$. Moreover, the observation of similar structural motifs when hydrophobic aromatic moieties are introduced, either via modification (SOMAmers) or as the nucleobases of UBPs, suggests that common themes, and perhaps even rules that will facilitate design, are possible.

Two examples where modified or wholly unnatural DNA was propagated within living cells have been reported, and the further optimization and use of the resulting semisynthetic organisms seems imminent. The next step towards retrieving information encoded by UBPs is the in vivo transcription of RNA containing unnatural nucleotides, and work towards this goal is in progress. UBPs have already been used for protein 
translation in vitro $[47,48]$ and their use in vivo represents the final step of creating a fully functional semi-synthetic organism capable of possessing and evolving new and useful attributes or functions, for example, the production of novel proteins for development as therapeutics. The substrate repertoires of the polymerases clearly extend beyond their natural substrates, and the synthesis of novel nucleotides, increasingly augmented with genetics and directed polymerase evolution, is enabling extension of the in vivo and in vitro applications of DNA and RNA and a dramatic increase in the potential of these already remarkable molecules.

\section{Acknowledgements}

FER acknowledges financial support from the National Institutes of General Medical Sciences (GM060005 and GM097489) and the Defense Advanced Research Projects Agency Folded Non-Natural Polymers with Biological Function Fold F(x) Program (Award No. N66001-14-2-4052). Any opinions, findings, and conclusions or recommendations expressed in this publication are those of the authors and do not necessarily reflect the views of DARPA.

\section{References and recommended reading}

Papers of particular interest, published within the period of review, have been highlighted as:

- of special interest

•- of outstanding interest

1. Diafa S, Hollenstein M: Generation of aptamers with an expanded chemical repertoire. Molecules 2015, 20:16643-16671. 
2. Mi J, Liu Y, Rabbani ZN, Yang Z, Urban JH, Sullenger BA, Clary BM: In vivo selection of tumor-targeting RNA motifs. Nat Chem Biol 2010, 6:22-24.

3. Cheng C, Chen YH, Lennox KA, Behlke MA, Davidson BL: In vivo SELEX for identification of bain-penetrating aptamers. Mol Ther Nucleic Acids 2013, 2:e67.

4. Gong Q, Wang J, Ahmad KM, Csordas AT, Zhou J, Nie J, Stewart R, Thomson JA, Rossi JJ, Soh HT: Selection strategy to generate aptamer pairs that bind to distinct sites on protein targets. Anal Chem 2012, 84:5365-5371.

5. Burmeister PE, Lewis SD, Silva RF, Preiss JR, Horwitz LR, Pendergrast PS, McCauley TG, Kurz JC, Epstein DM, Wilson C, Keefe AD: Direct in vitro selection of a 2'-O-methyl aptamer to VEGF. Chem Biol 2005, 12:25-33.

6. Friedman AD, Kim D, Liu R: Highly stable aptamers selected from a 2'-fully modified fGmH RNA library for targeting biomaterials. Biomaterials 2015, 36:110-123.

7. Ibach J, Dietrich L, Koopmans KR, Nobel N, Skoupi M, Brakmann S: Identification of a T7 RNA polymerase variant that permits the enzymatic synthesis of fully 2'-O-methyl-modified RNA. J Biotechnol 2013, 167:287-295.

8. Meyer AJ, Garry DJ, Hall B, Byrom MM, McDonald HG, Yang X, Yin YW, Ellington AD: Transcription yield of fully 2'-modified RNA can be increased 
by the addition of thermostabilizing mutations to T7 RNA polymerase mutants. Nucleic Acids Res 2015, 43:7480-7488.

9. Zhu B, Hernandez A, Tan M, Wollenhaupt J, Tabor S, Richardson CC: Synthesis of 2'-fluoro RNA by Syn5 RNA polymerase. Nucleic Acids Res 2015, 43:e94.

10. Hagiwara K, Fujita H, Kasahara $\mathrm{Y}$, Irisawa $\mathrm{Y}$, Obika S, Kuwahara M: In vitro selection of DNA-based aptamers that exhibit RNA-like conformations using a chimeric oligonucleotide library that contains two different xenonucleic acids. Mol Biosyst 2015, 11:71-76.

11. Chen T, Hongdilokkul N, Liu Z, Adhikary R, Tsuen SS, Romesberg FE: Evolution of thermophilic DNA polymerases for the recognition and amplification of C2'-modified DNA. Nat Chem 2016, doi:10.1038/nchem.2493.

12. MacPherson IS, Temme JS, Habeshian S, Felczak K, Pankiewicz K, Hedstrom L, Krauss IJ: Multivalent glycocluster design through directed evolution. Angew Chem Int Ed 2011, 50:11238-11242.

13. Horiya S, MacPherson IS, Krauss IJ: Recent strategies targeting HIV glycans in vaccine design. Nat Chem Biol 2014, 10:990-999.

14. Yu H, Zhang S, Chaput JC: Darwinian evolution of an alternative genetic system provides support for TNA as an RNA progenitor. Nat Chem 2012, 4:183-187. 
15. Kasahara Y, Irisawa Y, Fujita H, Yahara A, Ozaki H, Obika S, Kuwahara M: Capillary electrophoresis-systematic evolution of ligands by exponential enrichment selection of base- and sugar-modified DNA aptamers: target binding dominated by 2'-0,4'-C-methylene-bridged/locked nucleic acid primer. Anal Chem 2013, 85:4961-4967.

16. Kuwahara M, Obika S: In vitro selection of BNA (LNA) aptamers. Artif DNA PNA XNA 2013, 4:39-48.

-17. Pinheiro VB, Taylor Al, Cozens C, Abramov M, Renders M, Zhang S, Chaput JC, Wengel J, Peak-Chew SY, McLaughlin SH, Herdewijn P et al: Synthetic genetic polymers capable of heredity and evolution. Science 2012, 336:341-344.

This article describes the directed evolution of DNA polymerases that better recognize highly modified sugars such as HNA and FANA, which the authors use to evolve stabilized aptamers.

18. Alves Ferreira-Bravo I, Cozens C, Holliger P, DeStefano JJ: Selection of 2'deoxy-2'-fluoroarabinonucleotide (FANA) aptamers that bind HIV-1 reverse transcriptase with picomolar affinity. Nucleic Acids Res 2015, 43:9587-9599.

19. Taylor AI, Pinheiro VB, Smola MJ, Morgunov AS, Peak-Chew S, Cozens C, Weeks KM, Herdewijn P, Holliger P: Catalysts from synthetic genetic polymers. Nature 2015, 518:427-430. 
20. Hottin A, Marx A: Structural insights into the processing of nucleobasemodified nucleotides by DNA polymerases. Acc Chem Res 2016, 49:418-427.

•21. Gelinas AD, Davies DR, Janjic N: Embracing proteins: structural themes in aptamer-protein complexes. Curr Opin Struct Biol 2016, 36:122-132.

This review describes the structural analysis of three SOMAmers, which reveals that the appended hydrophobic aromatic rings are capable of creating protein-like hydrophobic clusters that mediate target recognition and folding.

•22. Tolle F, Brandle GM, Matzner D, Mayer G: A versatile approach towards nucleobase-modified aptamers. Angew Chem Int Ed 2015, 54:10971-10974. The authors demonstrate that C5-ethynyl-dU may be incorporated via PCR, and then its ethynyl groups may be modified via Click chemistry to allow access to a wide range of modified oligonucleotides.

23. Davies DR, Gelinas AD, Zhang C, Rohloff JC, Carter JD, O'Connell D, Waugh SM, Wolk SK, Mayfield WS, Burgin AB, Edwards TE et al: Unique motifs and hydrophobic interactions shape the binding of modified DNA ligands to protein targets. Proc Natl Acad Sci USA 2012, 109:19971-19976.

24. Gelinas AD, Davies DR, Edwards TE, Rohloff JC, Carter JD, Zhang C, Gupta S, Ishikawa Y, Hirota M, Nakaishi Y, Jarvis TC et al: Crystal structure of interleukin-6 in complex with a modified nucleic acid ligand. $J$ Biol Chem 2014, 289:8720-8734. 
25. Jarvis TC, Davies DR, Hisaminato A, Resnicow DI, Gupta S, Waugh SM, Nagabukuro A, Wadatsu T, Hishigaki H, Gawande B, Zhang C et al: Non-helical dna triplex forms a unique aptamer scaffold for high affinity recognition of nerve growth factor. Structure 2015, 23:1293-1304.

26. Malyshev DA, Romesberg FE: The expanded genetic alphabet. Angew Chem Int Ed 2015, 54:11930-11944.

27. Hirao I: Synthetic genetic polymers functioning to store and propagate information by genetic alphabet expansion. In: Reviews in Cell Biology and Molecular Medicine. Wiley-VCH Verlag GmbH \& Co. KGaA, (2006).

28. Benner SA, Chen F, Yang Z: Synthetic biology, tinkering biology, and artificial biology: A perspective from chemistry. In: Chemical Synthetic Biology. John Wiley \& Sons, Ltd, (2011):69-106.

29. Betz K, Malyshev DA, Lavergne T, Welte W, Diederichs K, Dwyer TJ, Ordoukhanian P, Romesberg FE, Marx A: KlenTaq polymerase replicates unnatural base pairs by inducing a Watson-Crick geometry. Nat Chem Biol 2012, 8:612-614.

30. Matsuda S, Fillo JD, Henry AA, Rai P, Wilkens SJ, Dwyer TJ, Geierstanger BH, Wemmer DE, Schultz PG, Spraggon G, Romesberg FE: Efforts toward expansion of the genetic alphabet: structure and replication of unnatural base pairs. J Am Chem Soc 2007, 129:10466-10473. 
31. Johar Z, Zahn A, Leumann CJ, Jaun B: Solution structure of a DNA duplex containing a biphenyl pair. Chemistry 2008, 14:1080-1086.

32. Malinovskii VL, Wenger D, Haner R: Nucleic acid-guided assembly of aromatic chromophores. Chem Soc Rev 2010, 39:410-422.

33. Teo YN, Kool ET: DNA-multichromophore systems. Chem Rev 2012, 112:4221-4245.

-34. Kimoto M, Yamashige R, Matsunaga K, Yokoyama S, Hirao I: Generation of high-affinity DNA aptamers using an expanded genetic alphabet. Nat Biotechnol 2013, 31:453-457.

This article describes one of the first two examples in which an expanded genetic alphabet was used in evolution, in this case to evolve aptamers that bind VEGF-165 or IFN-@).

35. Zhang L, Yang Z, Sefah K, Bradley KM, Hoshika S, Kim MJ, Kim HJ, Zhu G, Jimenez E, Cansiz S, Teng IT et al: Evolution of functional six-nucleotide DNA. J Am Chem Soc 2015, 137:6734-6737.

-36. Sefah K, Yang Z, Bradley KM, Hoshika S, Jimenez E, Zhang L, Zhu G, Shanker S, Yu F, Turek D, Tan $\mathrm{W}$ et al: In vitro selection with artificial expanded genetic information systems. Proc Natl Acad Sci USA 2014, 111:1449-1454.

This article describes one of the first two examples where an expanded genetic alphabet was used in evolution, in this case to evolve aptamers that bind breast or liver cancer cells. 
37. Kimoto M, Hikida Y, Hirao I: Site-specific functional labeling of nucleic acids by in vitro replication and transcription using unnatural base pair systems. Israel Journal of Chemistry 2013, 53:450-468.

38. Li Z, Lavergne T, Malyshev DA, Zimmermann J, Adhikary R, Dhami K, Ordoukhanian P, Sun Z, Xiang J, Romesberg FE: Site-specifically arraying small molecules or proteins on DNA using an expanded genetic alphabet. Chemistry 2013, 19:14205-14209.

39. Lavergne T, Lamichhane R, Malyshev DA, Li Z, Li L, Sperling E, Williamson JR, Millar DP, Romesberg FE: FRET characterization of complex conformational changes in a large 16s ribosomal RNA fragment site-specifically labeled using unnatural base pairs. ACS Chem Biol 2016, 10.1021/acschembio. $5 b 00952$.

40. Krueger AT, Peterson LW, Chelliserry J, Kleinbaum DJ, Kool ET: Encoding phenotype in bacteria with an alternative genetic set. J Am Chem Soc 2011, 133:18447-18451.

41. Pezo V, Liu FW, Abramov M, Froeyen M, Herdewijn P, Marliere P: Binary genetic cassettes for selecting XNA-templated DNA synthesis in vivo. Angew Chem Int Ed 2013, 52:8139-8143. 
42. Maruyama H, Furukawa K, Kamiya H, Minakawa N, Matsuda A: Transcription of 4'-thioDNA templates to natural RNA in vitro and in mammalian cells. Chem Commun (Camb) 2015, 51:7887-7890.

•43. Marliere P, Patrouix J, Doring V, Herdewijn P, Tricot S, Cruveiller S, Bouzon M, Mutzel R: Chemical evolution of a bacterium's genome. Angew Chem Int Ed 2011, 50:7109-7114.

This article reports the creation of an $E$. coli cell wherein dT has been mostly replaced by its 5-chlorouracil analog and thus represents the first semi-synthetic organism with an altered genetic alphabet.

•.44. Malyshev DA, Dhami K, Lavergne T, Chen T, Dai N, Foster JM, Correa IR, Jr., Romesberg FE: A semi-synthetic organism with an expanded genetic alphabet. Nature 2014, 509:385-388.

This article reports the creation of $E$. coli cell that stably propagates within its DNA an unnatural base pair and thus represents the first semi-synthetic organism with an expanded genetic alphabet.

45. Anosova I, Kowal EA, Dunn MR, Chaput JC, Van Horn WD, Egli M: The structural diversity of artificial genetic polymers. Nucleic Acids Res 2016, 44:1007-1021.

46. Rohloff JC, Gelinas AD, Jarvis TC, Ochsner UA, Schneider DJ, Gold L, Janjic N: Nucleic acid ligands with protein-like side chains: modified aptamers and their use as diagnostic and therapeutic agents. Mol Ther Nucleic Acids 2014, 3:e201. 
47. Bain JD, Switzer C, Chamberlin AR, Benner SA: Ribosome-mediated incorporation of a nonstandard amino-acid into a peptide through expansion of the genetic-code. Nature 1992, 356:537-539.

48. Hirao I, Ohtsuki T, Fujiwara T, Mitsui T, Yokogawa T, Okuni T, Nakayama H, Takio K, Yabuki T, Kigawa T, Kodama $\mathrm{K}$ et al: An unnatural base pair for incorporating amino acid analogs into proteins. Nat Biotechnol 2002, 20:177182. 


\section{Figure captions}

Figure 1. Evolution of nucleic acids via SELEX. (a) RNA SELEX. (b) DNA SELEX. In both RNA and DNA SELEX, larger libraries of oligonucleotides (up to $\sim 10^{15}$ ) with different sequences are subjected to selection for a desired function (binding or catalysis), and the recovered oligonucleotides are then amplified and subjected to additional rounds of selection.

Figure 2. Modified nucleotides used in SELEX. (a) Chemical structures of ANA, FANA, TNA, LNA, HNA, and CeNA. (b) Position and structure of common nucleobase modifications. Sugar and phosphates omitted for clarity. The five common classes of linkers are shown with additional and variable functionality indicated with R'.

Figure 3. Structures of SOMAmers bound to their respective target proteins, including an expanded view of each hydrophobic cluster. (a, d) PDGF SOMAmer (PDB ID 4HQU). (b, e) IL-6 SOMAmer (PDB ID 4NI9). (c, f) NGF SOMAmer (PDB ID 4ZBN).

Figure 4. UBPs that may be replicated and transcribed by native polymerases in vitro. (a) Chemical structures (sugar and phosphates omitted for clarity). (b) Structure of an early progenitor of the d5SICS-dNaM UBP [30]. (c) Structure of d5SICS-dNaM in duplex DNA [29]. (d) Structure of d5SICS-dNaM as formed in the active site of a DNA polymerase (PDB ID 3SV3).

Figure 5. E. coli based semi-synthetic organism. Triphosphates provided in the growth medium diffuse into the periplasm (likely via porins) and then are transported into the 
cytoplasm by a nucleoside triphosphate transporter where they are used to replicate DNA containing the d5SICS-dNaM UBP. 
Figure 1

(a)

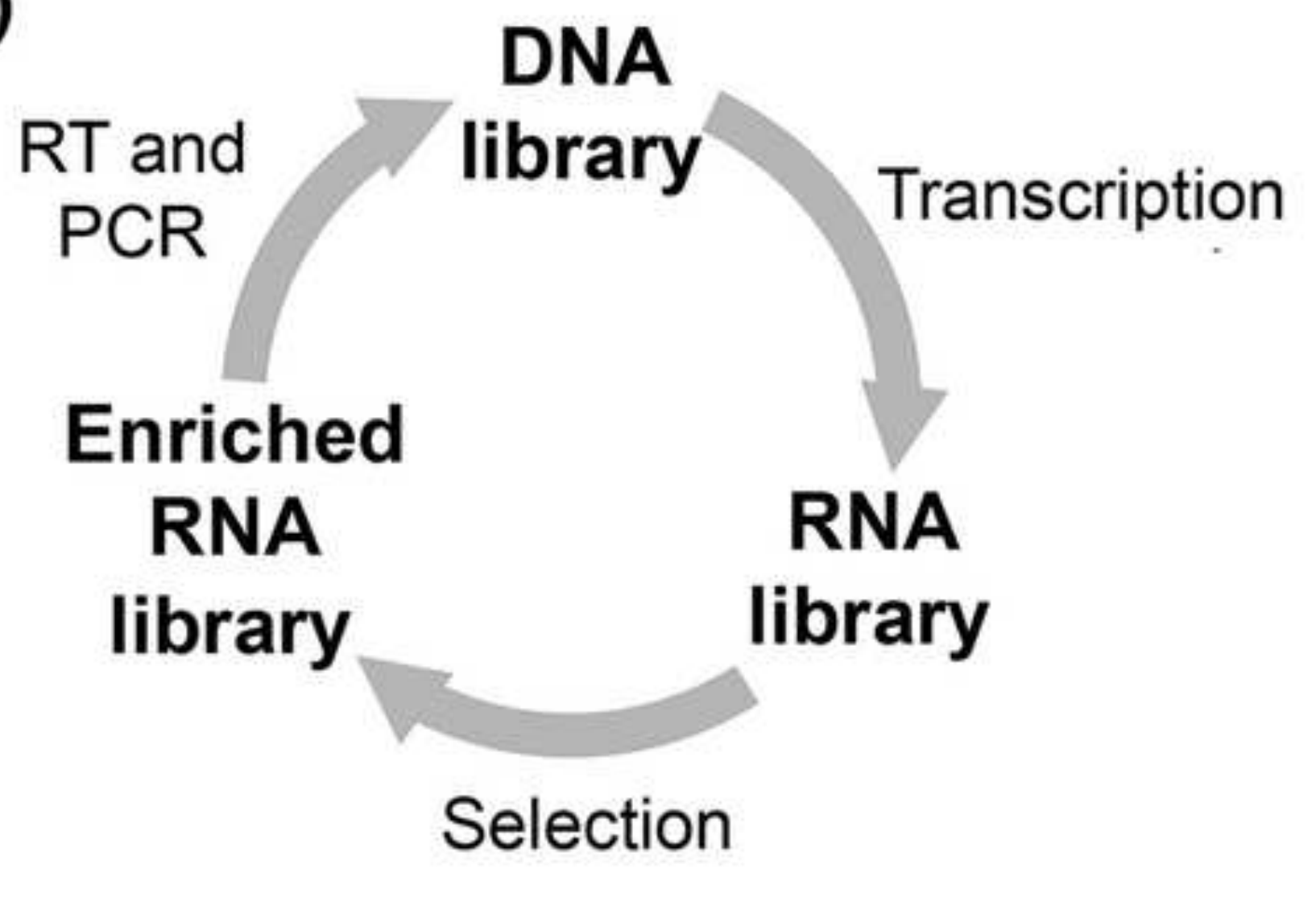

Selection

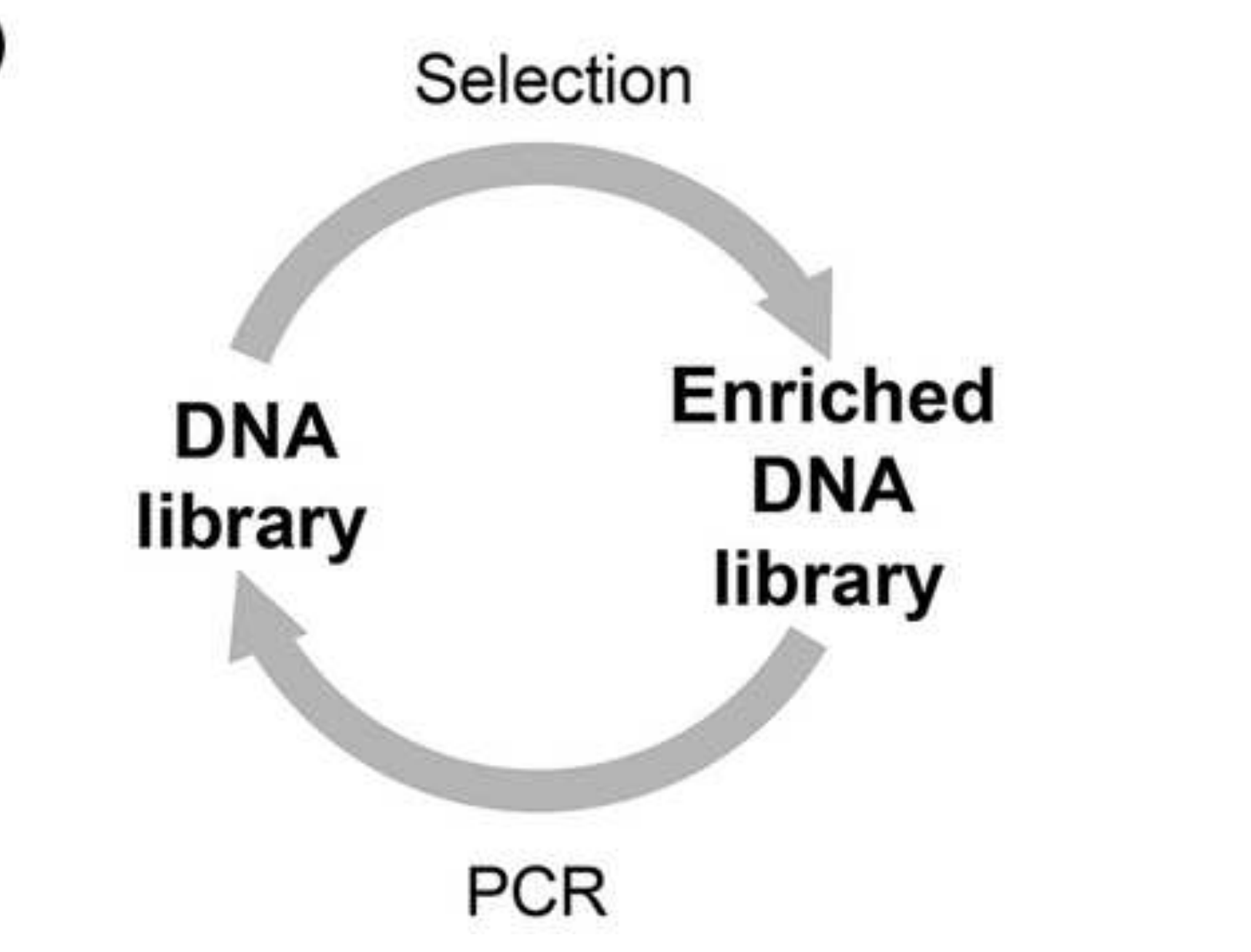

\section{DNA}

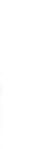
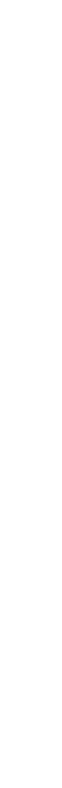

.
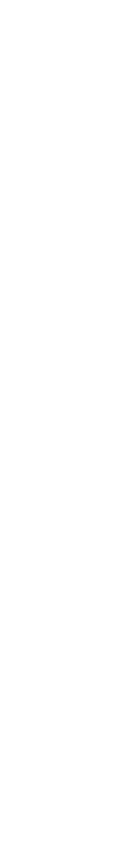

\section{(b)}


(a)

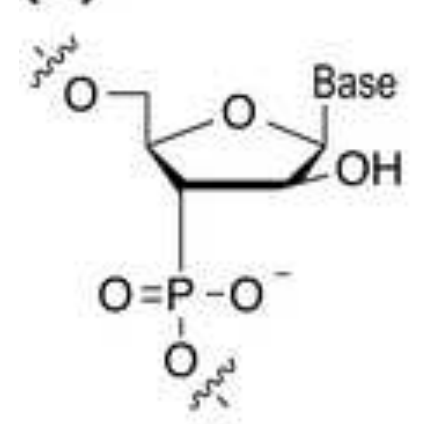

ANA

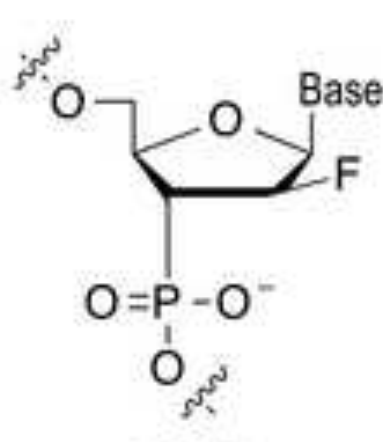

FANA

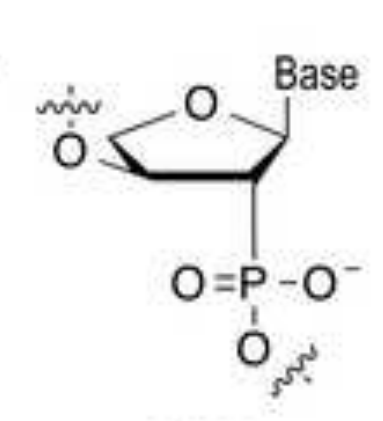

TNA

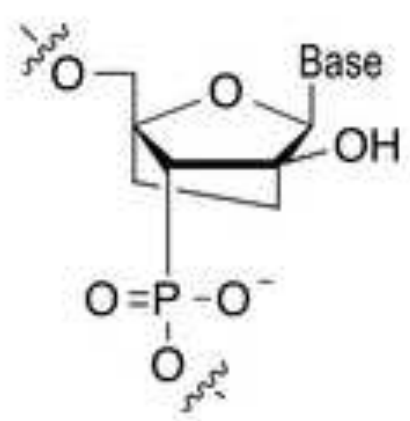

LNA

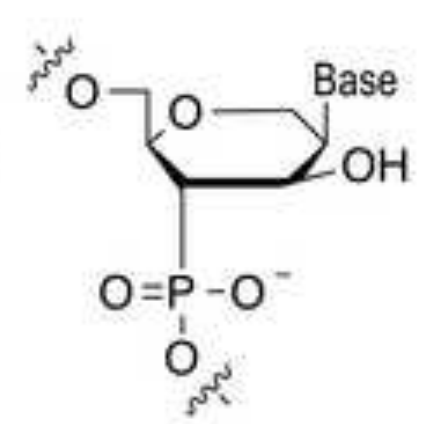

HNA

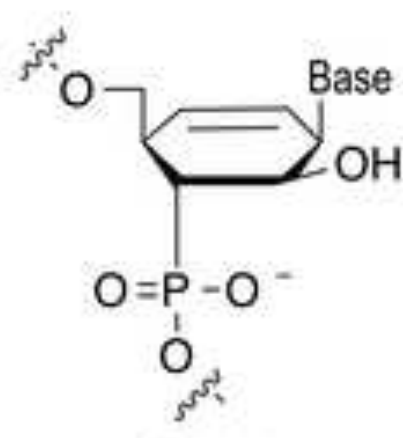

CeNA

(b)<smiles>[2H]c1cn(C)c(=O)[nH]c1=O</smiles>

U/dT<smiles>[R]c1cn(N)c2ncnc(N)c12</smiles>

(d) A

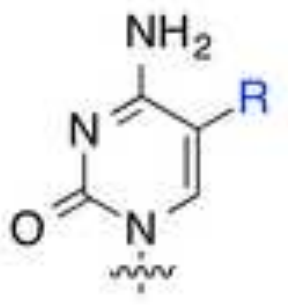

(d)C<smiles>[R]c1cn(C)c2nc(N)[nH]c(=O)c12</smiles>

(d) G
Linker (R)
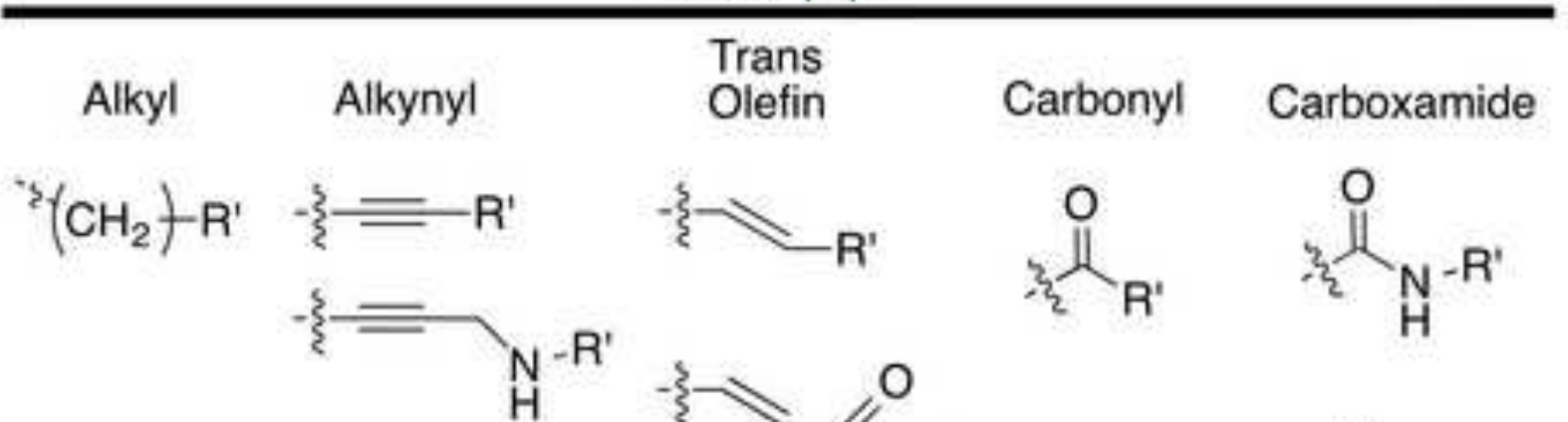<smiles>[Z]C([Y])=O</smiles><smiles>[Y6]NC(=O)C([Y6])=O</smiles><smiles>[Z20]NC([R7])=O</smiles>

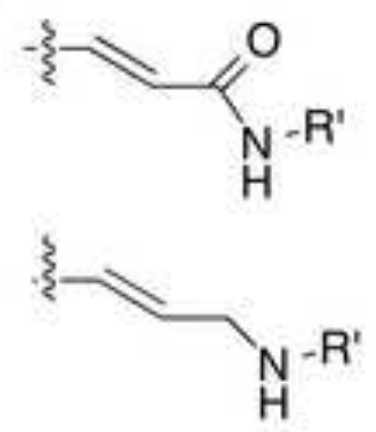

$\pi_{\mathrm{O}}^{\mathrm{N}} \pi_{\mathrm{R}^{\prime}}^{\mathrm{H}}$ 
(a)

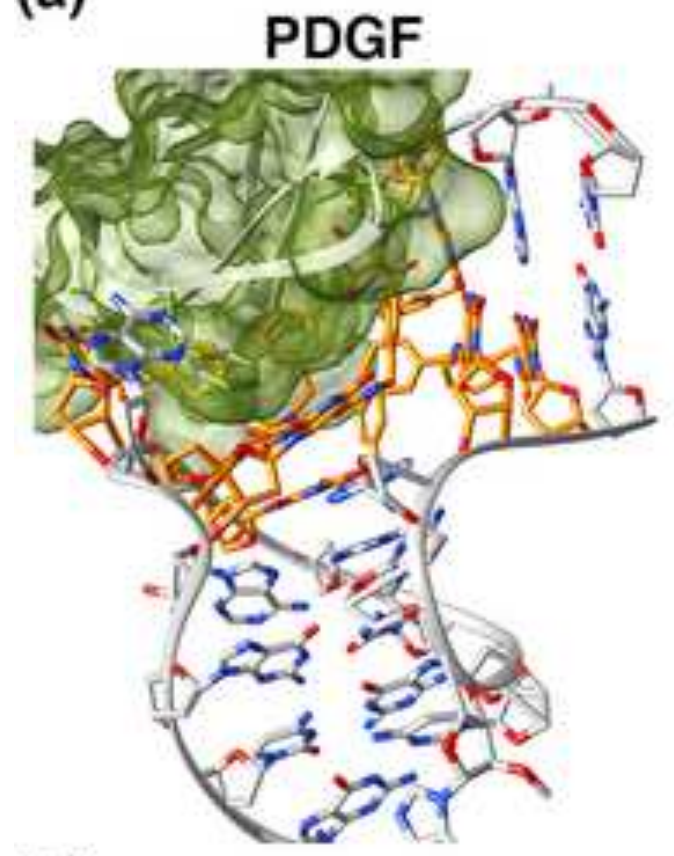

(d)

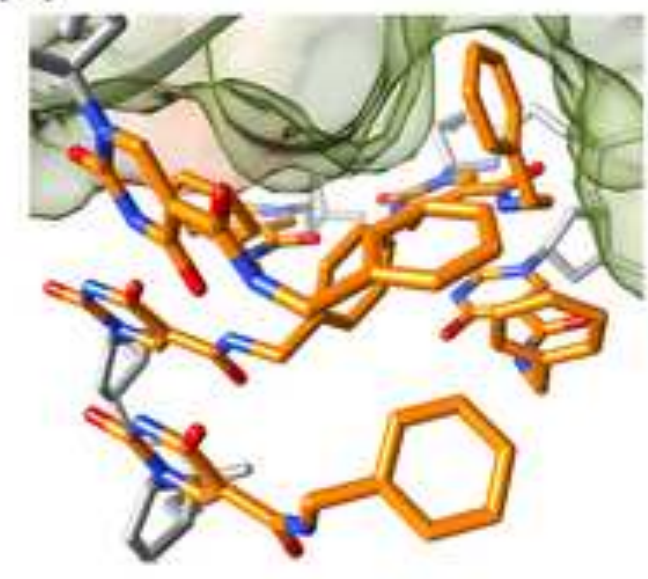

(b)

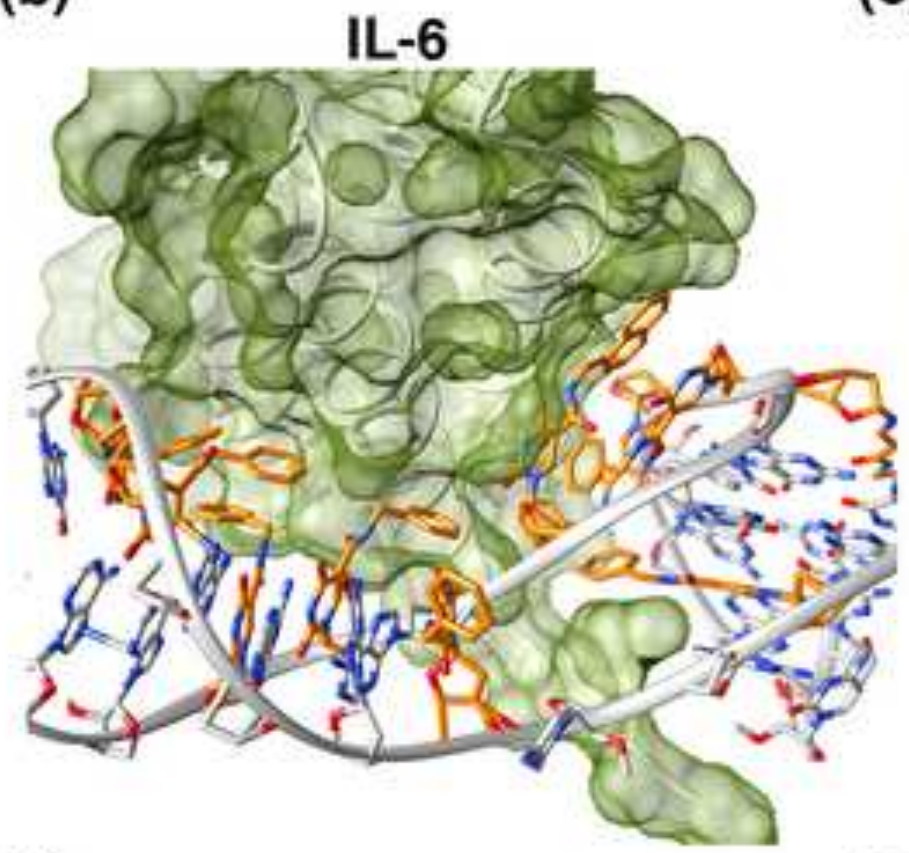

(e)

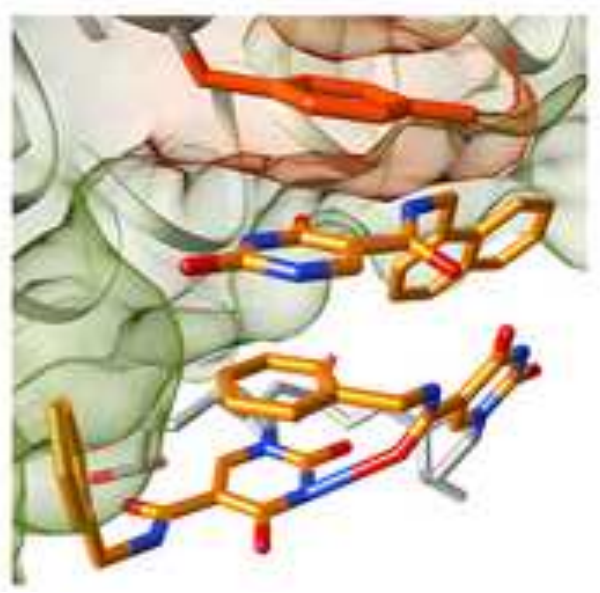

(c)

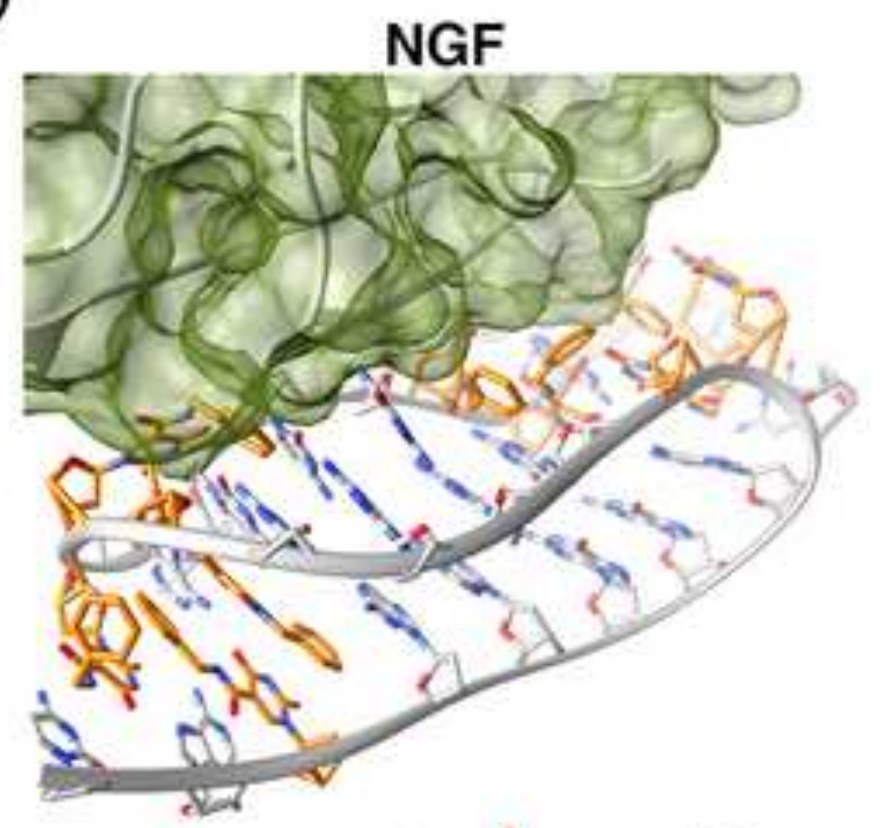

(f)
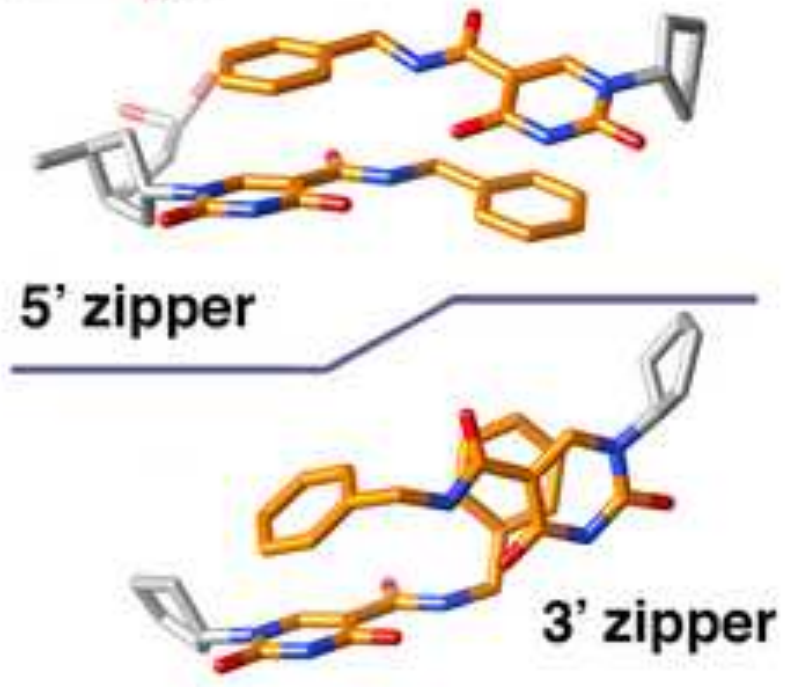
(a)

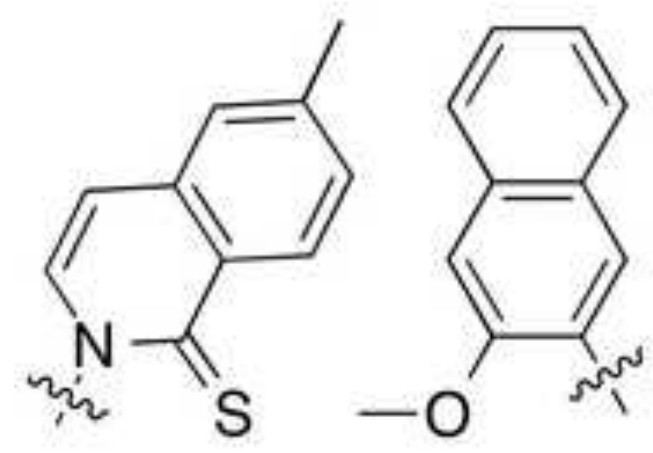

d5SICS-dNaM

(b)

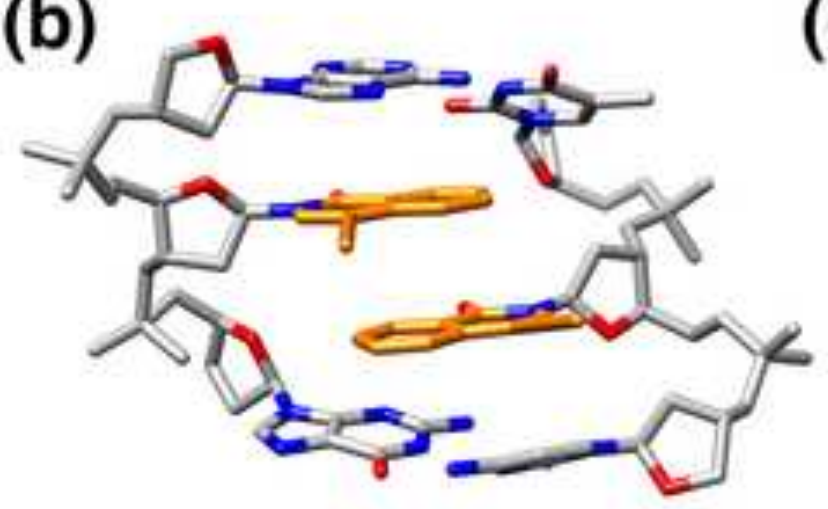

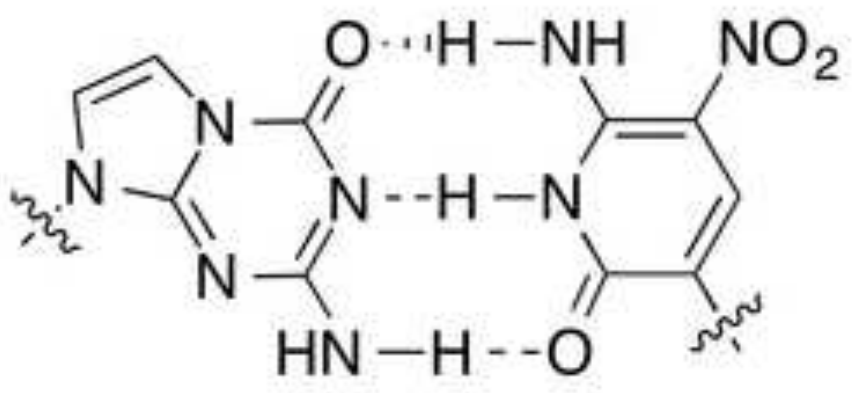

$d P-d Z$

(c)

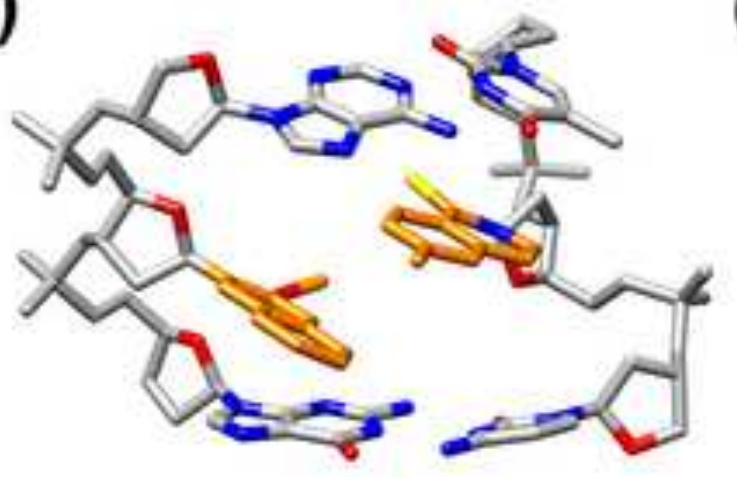

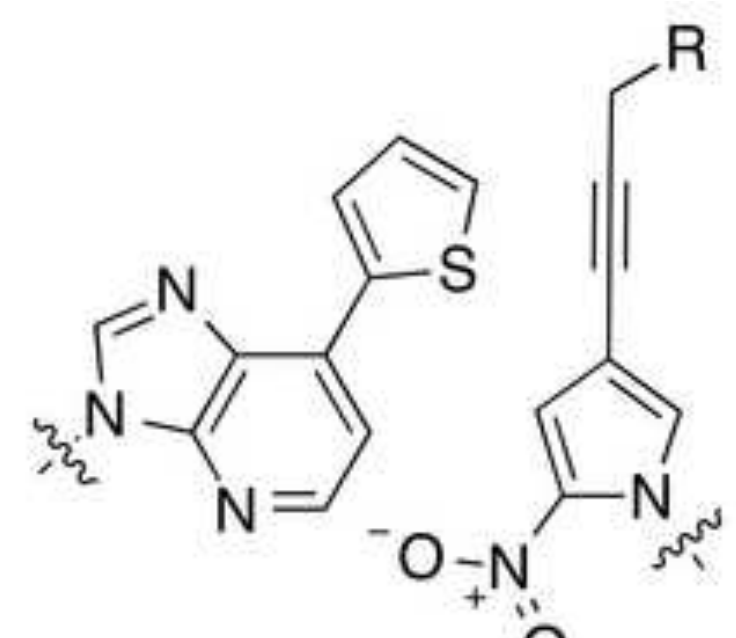

dDs-dPx O

(d)

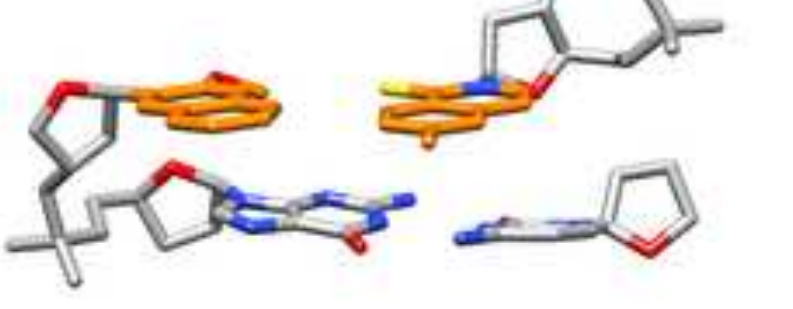




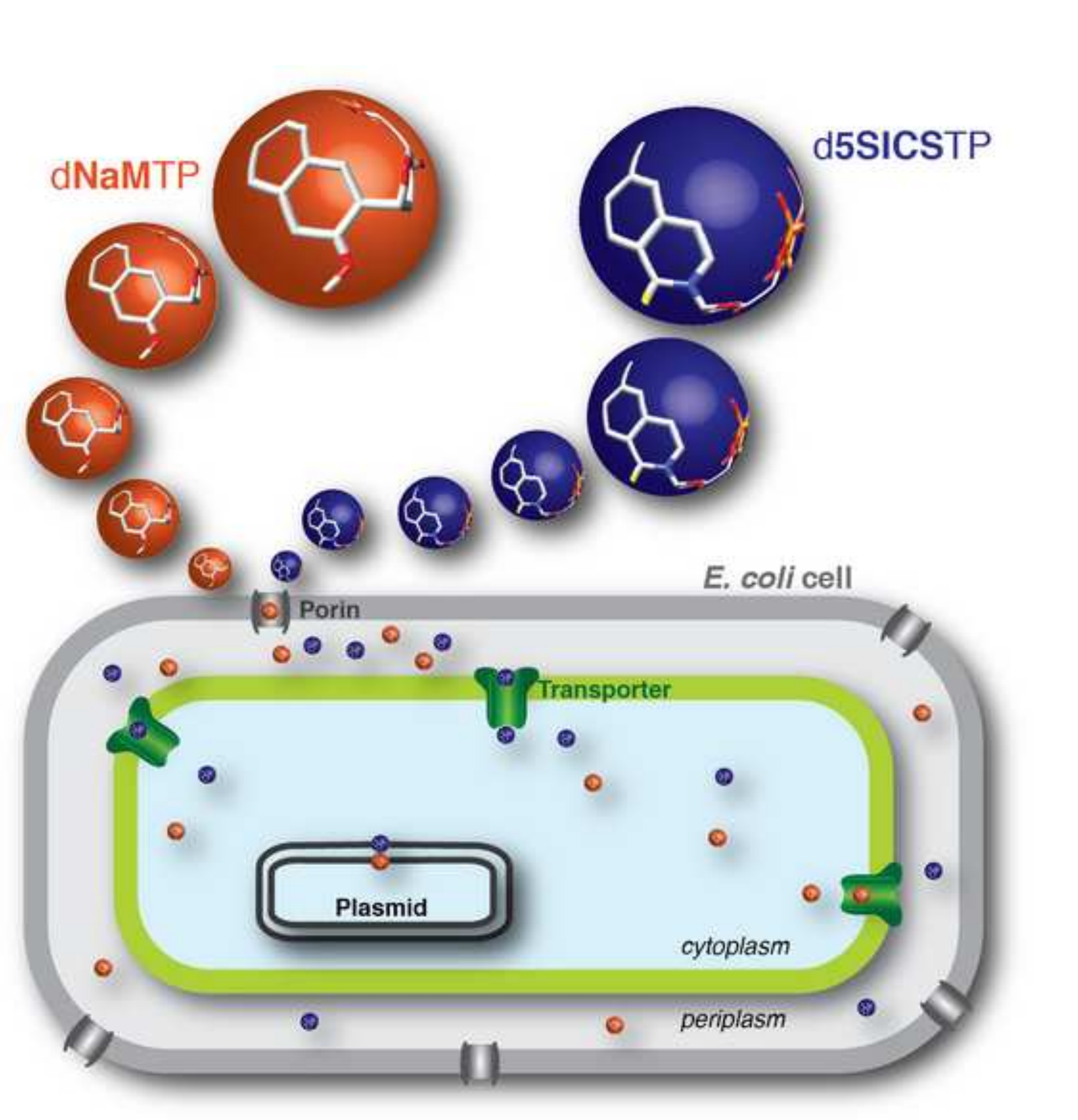

Figure 5
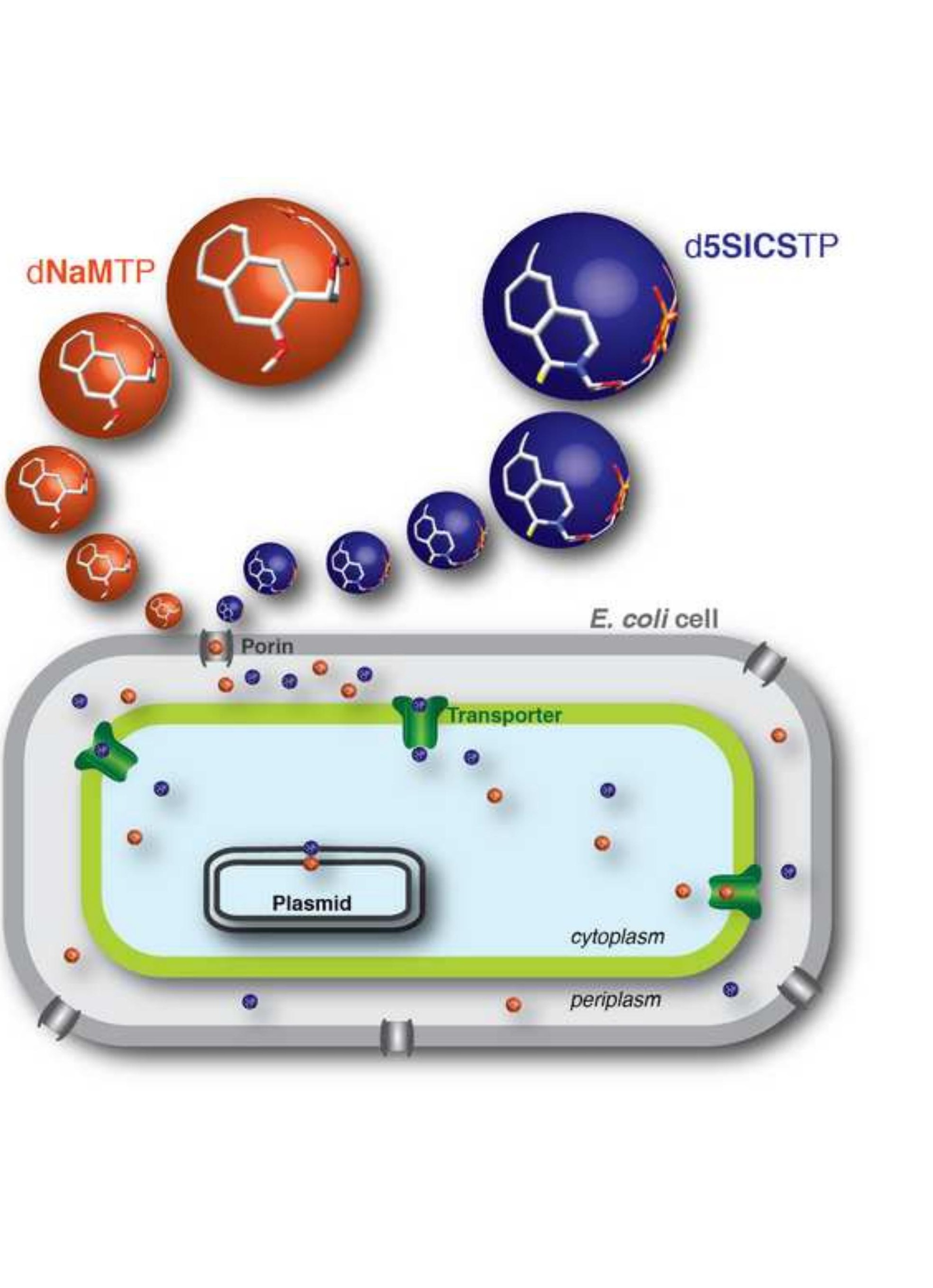

\section{d5SICSTP}

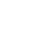


Graphical Abstract

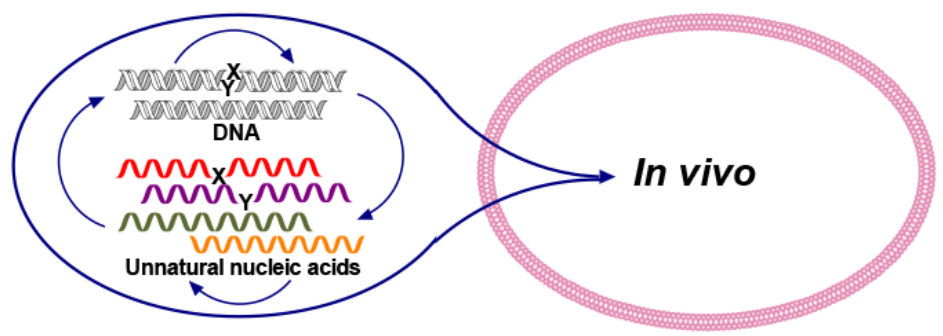

